\title{
Evaluation of pseudo-haptic feedback for simulating torque: a comparison between isometric and elastic input devices
}

\author{
Alexis Paljic* \\ Jean-Marie Burkhardt ${ }^{\dagger}$ \\ Sabine Coquillart* \\ *INRIA, i3D Group \\ ${ }^{\dagger}$ INRIA, Eiffel Group \\ Institut National de Recherche en Informatique et Automatique \\ Domaine de Voluceau 78153 Le Chesnay Cedex France \\ \{Alexis.Paljic, Jean-Marie.Burkhardt, Sabine.Coquillart\}@inria.fr
}

\begin{abstract}
In this work, we investigate whether pseudo-haptic feedback is suitable for simulating torque feedback. Pseudohaptic feedback is based on the coupling of visual feedback and the internal resistance of an input device which passively reacts to the user's applied force. An experiment was conducted to evaluate this feedback and compare isometric and elastic input devices. It involved compliance discrimination between real torsion springs and pseudo-haptic simulated torsion springs. Results show that torque haptic feedback was successfully simulated, with a difference in performance between device types. The elastic device yielded better resolution but higher subjective distortion of perception compared to the isometric device. Results are discussed on the basis of user answers, answer time, and applied torque.
\end{abstract}

\section{Introduction}

Torque gives important information in everyday manipulations, such as opening bottles or turning a key. Mounting parts of a mechanism, or peg-in-a-hole tasks, often involve torque constraints on the object being manipulated. This is inherent to the complex nature of human movements which are a combination of translations and rotations, thus, force reaction of mechanically constrained objects is very likely to contain a torque component. Torque information can be computed in virtual environments to provide the user with force feedback. Studies show that force feedback can be a valuable technique in domains like medical simulation [6] or collaborative environments [5][17]. Torque feedback may be simulated with active devices. The 6dof Phantom [16][4], the delta haptic device [11], the Virtuose 6D [2] or the Freedom6s [3] can provide the user with torque feedback in addition to translational force feedback. A magnetic levitation haptic interface [7] offers six degrees of freedom force feedback. Some systems are dedicated to torque feedback, such as the Gyro Moment Haptic Interface [1] or a Hand-Held Torque Feedback Device by Fukui et al. [9]. Ho et al. proposed a ray-based haptic rendering [12] for force and torque feedback. Using a line probe and two Phantom devices, users were able to touch and feel virtual objects with a line segment model of the probe and perceive torque information, which is not possible with single point interaction.

Another way to provide haptic feedback is through the use of cross-modal interactions. Biocca et al. [8] defined these as "perceptual illusions in which users use sensory cues in one modality to fill in the missing components of perceptual experience." and studied how such techniques can improve a user's sense of presence. In their study, Biocca et al. list different cross-modal sensory interactions. Among them are cross modal transfer (or synesthesia) and cross-modal enhancement or modification. Synesthesia is an extreme case of cross modal interaction "in which sensory information of one sensory channel produces experiences in another unstimulated sensory channel that receives no apparent stimulation from the virtual environ$m e n t$ ". They report results from an experiment that supports the existence of visual/haptic synesthesia, which enhances the user's sense of presence. Pseudo haptic feedback, proposed by Lecuyer et al. [15] is a form of synesthesia. The authors defined pseudo-haptic systems as "systems providing haptic information generated, augmented or modified, by the influence of another sensory modality [14]. They reported a study showing its ability to feed back force cues. 
The background of this work is to extend the use of pseudo-haptic feedback to torque feedback and to investigate which of isometric or elastic input is the most suitable for this purpose. Our objective is to use torque pseudo-haptic feedback in an immersive virtual environment. To do so, there is a need to provide both theoreticallygrounded and empirically-validated rationales for the design of 3D interfaces. We mean to develop our knowledge of user-interface principles with which to develop a low cost pseudo-haptic device for use in an immersive environment.

The concept of pseudo haptic feedback requires that the passive device resist user-applied forces, thus it must have mechanical properties that allow this behavior. This can be implemented either by using an isometric device (with infinite stiffness and no displacement) or by using an elastic device (with a finite internal stiffness and displacement during manipulation).

The purpose of the user study presented in this paper is to evaluate the suitability of pseudo-haptic feedback for torque feedback and compare two passive isometric and elastic devices.

The paper begins with an overview of previous work in the field of pseudo-haptic feedback and torque and stiffness discrimination studies. It is followed by a description of the method, setup, and results of the experiment in Section 3 Results, based on psychophysical methods, are discussed in Section 4

\section{Previous Work}

Lecuyer et al. conducted an experiment to measure the possibility of providing the user with haptic information by means of a passive input device combined with visual feedback. The device used was the Spaceball, which was considered as isometric in [15] because of its small displacement, but in this paper we will consider it as elastic, according to the definition that we proposed in the Introduction. A task of stiffness discrimination between real and virtual springs was used in the study. Results showed that subjects were able to discriminate successfully between a virtual spring and a real one. Two parameters were computed to characterize the subjects' performance when using the device, based on the psychophysics tradition. The first of these is the Just Noticeable Difference (JND) which is the just detectable variation of intensity of a specific stimulus. It corresponds to the value of the stimulus (here, the pseudohaptic spring) for which $75 \%$ of users perceive a difference in relation to the reference spring. The second is the Point of Subjective Equality (PSE), the value of the virtual spring stiffness that is perceived as equal to the real spring stiffness. The authors found an average JND of $13.4 \%$. An illusion phenomenon was observed since the proprioceptive sense of the subjects was blurred by visual feedback.
To our knowledge, few studies on torque discrimination have been done. Jandura et al. [13] conducted experiments to characterize human performance in sensing and controlling torque. They found a JND of $12.7 \%$ when the reference torque was $60 \mathrm{mN} \cdot \mathrm{m}$. Our interest in the role of the type of input device (isometric or elastic) in pseudo-haptic simulation was raised by different studies. A study by Zhai [19] evaluated elastic versus isometric rate control devices (in opposition to position control devices) in a 3D object positioning task. He found that the richer proprioceptive feedback afforded by an elastic controller is necessary for achieving superior performance in the early stages of learning. Subjects performed equally well with the isometric controller in later learning stages. In another work, Tan et al. [18] conducted experiments on compliance (inverse of stiffness) discrimination in which work cues were eliminated and force cues minimized. The average JND for compliance ranged from $15 \%$ to $99 \%$ and was much larger than the average JND obtained from previous compliance discrimination experiments in which both work and terminal force cues were available to the subject. They concluded that manual resolution of compliance deteriorates when force and/or work cues are reduced or eliminated. These studies may support the hypothesis that pseudo-haptic feedback for torsion simulation could be better simulated with an elastic input device that would allow the user to have more proprioceptive information, as well as work and force cues, to help his perception. The next section presents the experiment which studies this hypothesis.

\section{Method}

To evaluate the proposed torque feedback and compare between isometric and elastic input, we chose a three-factor between subjects, repeated-measures design. Two groups of subjects went through a stiffness discrimination task between real torsion springs and virtual torsion springs that are pseudo-haptically simulated. For the first group the input device was isometric, and for the second group elastic. The independent factors were: 1) Type of device (isometric or elastic), 2) Real spring stiffness (two different real torsion springs were used), and 3) Virtual spring stiffness.

\subsection{Setup}

We built a prototype system that enables users to manually compare real torsion springs and pseudohaptic torsion springs (see Figure 11. The users were able to operate the system by manually turning plastic caps. Two caps are dedicated to real torsion spring manipulation and one is used to simulate a pseudo haptic torsion spring. The prototype presented in Figure 1 has one more cap, corresponding to a third real spring that was not used in the experiment. 


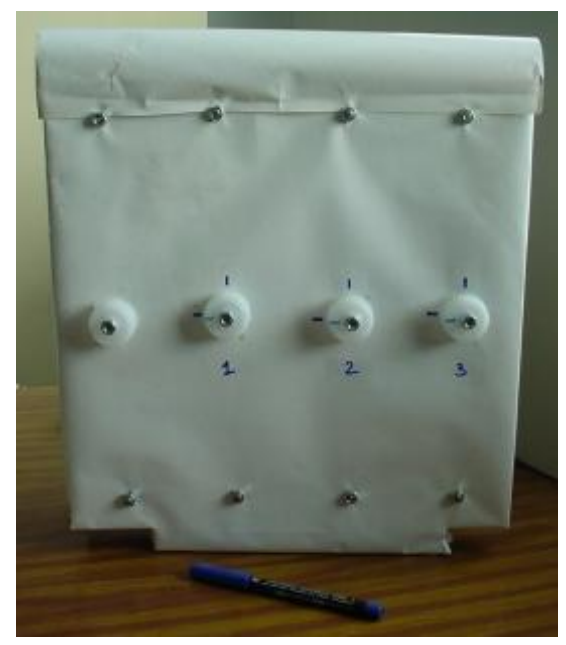

Figure 1. Front view of the experimental setup.

\subsubsection{Real Torsion springs}

Two real torsion springs were used in the experiment, known to the user as the "real springs". Users estimated the compliance of the real torsion springs by manually turning the plastic caps which offered resistance to rotation. This was done by embedding axles into the structure. On each axle, we fixed a plastic cap that the subjects could grip and turn. The rotational movement of each cap was constrained by springs fixed to the axle as shown in Figure 2 The cap has grooves on its edges to let the user grip it easily. Friction effects were minimized by applying a lubricant to the contact points between the axle and the structure. The torsion constant of each torsion spring was empirically derived: we measured the weight needed to rotate the cap through a given angle, given the cap diameter $(3.1 \mathrm{~cm})$. Values of the two torsion spring constants were $C 1=$ $2.0510^{-2} \mathrm{~N} \cdot \mathrm{m} \cdot \mathrm{rad}^{-1}$ and $C 2=3.210^{-2} \mathrm{~N} \cdot \mathrm{m} \cdot \mathrm{rad}^{-1}$. In the following, we will use the term "stiffness" to refer to "torsion constant". These two real springs were chosen to have stiffnesses that are representative of two common real-world situations: a weakly stiff spring (spring 1), and a medium stiff spring (spring 2). During preliminary experiments, we also chose the stiffness of spring 2 to avoid user fatigue (we limited maximum torque).

\subsubsection{Pseudo-haptic simulated torsion spring}

The pseudo haptic simulation consists of two elements: the passive input device and the visual simulation. The visual simulation represents a cap of the same size and aspect as the real ones (see Figure 3 ).

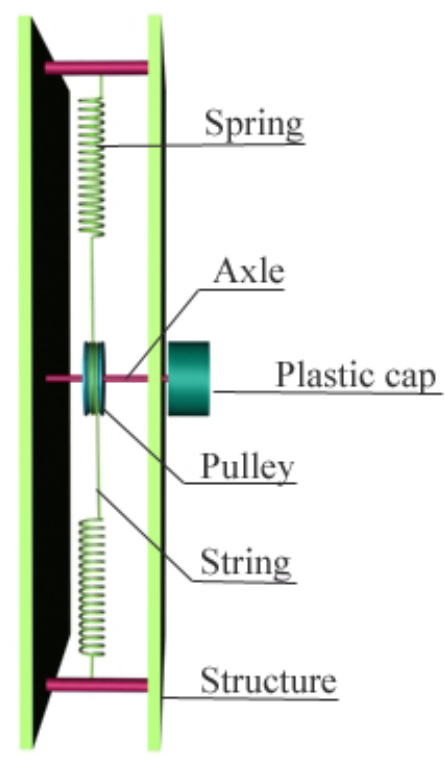

Figure 2. Real torsion spring assembly

Its rotational behavior is computed from the simulated virtual stiffness $C_{v}$. $C_{v}$ is simulated using Hooke's law (1). Given the torque applied by the user $\Gamma_{u}$ on the input device and the simulated $C_{v}$, the simulation sets the angular position of the virtual cap at $\theta_{v}$.

$$
\Gamma_{u}=C_{v} \cdot \theta_{v}
$$

The visual simulation runs on a Pentium III $800 \mathrm{MHz}$ PC, with a 21" CRT monitor monoscopic display, at a $50 \mathrm{~Hz}$ frame rate.

The passive input device uses one of the caps, coupled to a FlexiForce force sensitive resistor (FlexiForce ${ }^{\mathrm{TM}} \mathrm{ELF}^{\mathrm{TM}}$ system, Sensor B101-M). The FlexiForce sensor is connected to a PC workstation running the visual simulation. The isometric or elastic nature of this input device is a key issue of this work.

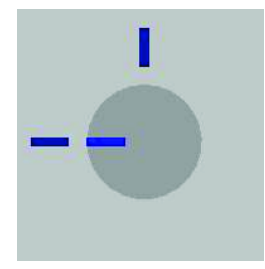

Figure 3. Visual feedback of the virtual torsion spring 


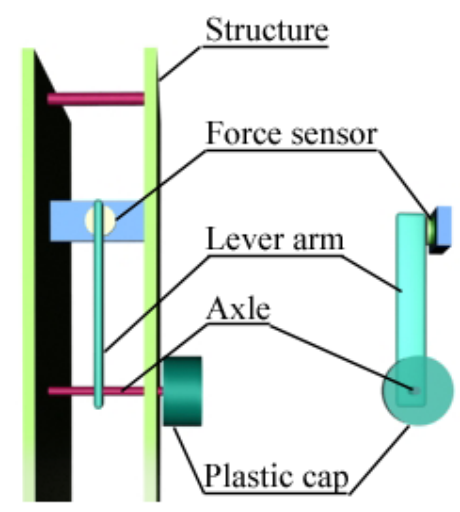

Side view

Front view

Figure 4. Isometric input device assembly. The cap cannot move, the force sensor is placed on a fixed plate, and a lever arm presses on it.

Isometric input device: The isometric input device is realized with a static cap so its real stiffness is infinite. The cap is connected to the FlexiForce sensor as shown in Figure 4 By isometric we mean that the device itself is not moving, however the user's fingers can be deformed during manipulation. The device was calibrated with a maximum $7 \%$ error. The group that did the experiment with the isometric device was made up of 17 people without known visual and haptic impairments. 5 had corrected vision. There were 3 women and 14 men, aged from 26 to 53. 2 were left-handed and 15 were right-handed.

Elastic input device: The elastic input device was realized using a real spring and by embedding the force sensor as shown in Figure 5. It had a finite internal stiffness and allowed rotation of the cap during displacement. The derived torsion constant of the device was $C=9.96 \cdot 10^{-2} \mathrm{~N} \cdot \mathrm{m} \cdot \mathrm{rad}^{-1}$. The device was calibrated with a maximum $6 \%$ error. The group that did the experiment with the elastic device was made up of 2 women and 15 men. All of them were right handed, they were aged from 25 to 33, and all had normal or corrected vision.

\subsection{Procedure}

The method used was a constant stimuli method with a two-alternative forced choice. We carried out the experiment with more subjects (17 subjects in each group) than in the usual psychophysics studies ( 3 or 4 subjects), in order to evaluate the ability of this setup to give torque feedback to a wider variety of users. However, to reduce user fatigue, we

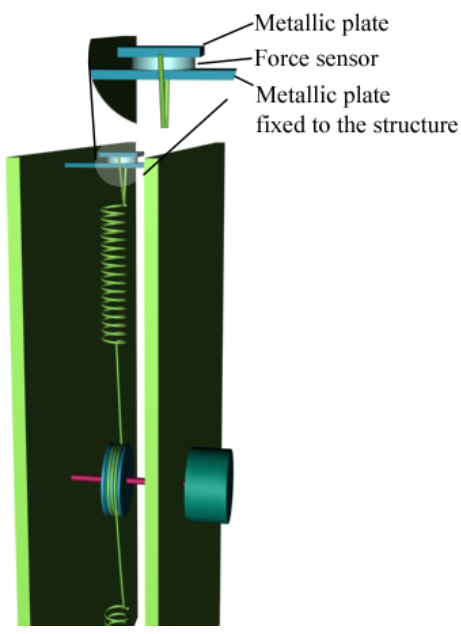

Figure 5. Elastic input device assembly. The device's cap rotation is elastic thanks to a real spring and the force sensor is placed so a metallic plate presses on it when torque is applied to the device.

used a smaller number of trials. Subjects were presented with a set of 32 experimental comparison trials. Two real springs were used (numbered 1 and 2 on the setup). The presented virtual spring had a virtual stiffness that was chosen from among 8 values of $-40 \%,-30 \%,-20 \%,-10 \%, 10 \%$, $20 \%, 30 \%$, and $40 \%$ of the presented real spring stiffness. Each case was presented twice, yielding 32 comparisons (2 repetitions $\times 2$ real springs $\times 8$ virtual springs), whose order of appearance was randomly shuffled. The users sat in front of the computer screen, with the experimental system within the reach of their dominant hand. Much attention was paid to ensure that each torsion spring, real or virtual, was held with the same finger, arm and body configuration throughout the test. Subjects placed their forearms on a pile of paper that raised the hand to the height of the caps. For each test the person had to decide "which of the real or virtual springs is the stiffer?" and typed the answer on the keyboard. Subjects were asked to look at the real spring when manipulating it, and to look at the visual feedback when testing the virtual spring. A maximum turning limit was indicated on both real and virtual springs at $90^{\circ}$. A cardboard cover was placed over the input device (virtual spring) so the subjects could not see the cap while turning it. Subjects were asked to start each test by real spring manipulation. They could switch between real and virtual springs without restriction. No information about the exactness of the answers was given during the tests. In order to familiarize the subjects with the task, each subject had ten trial tests before the beginning of the experiment. 


\subsection{Gathered data}

- Answer. The answer for each real/virtual spring comparison was recorded.

- Applied Torque. The value of the torque applied on the device during the tests was stored for each user.

- Answer time. Time elapsed between the presentation of the comparison spring and the answer.

- Type of strategy. The subjects were also asked to describe their strategy for discrimination, if any.

- Perceived displacement of the device. At the end of the tests the subjects were asked to draw the estimated angle of rotation of the plastic cap used on the input device. We insisted that this be the physical rotation and not the visual feedback.

\subsection{Results}

Just Noticeable Difference, Point of Subjective Equality. The analysis of answers follows the usual method described in [10] to compute the JND and PSE ${ }^{1}$. All subjects' answers were added together and used to trace psychometric functions for each real torsion spring. The psychometric function represents the proportion of "virtual spring is stiffer" answers, as a function of the comparison (virtual) stiffness. We assumed that this function corresponds to a normal distribution of 0 average and a standard deviation equal to 1 . Under this assumption, Z-score transformation of the proportion of "virtual spring is stiffer" answers yields a linear function (see Figure 8). Usual parameters (JND, PSE) are calculated using a linear regression of the $\mathrm{Z}$-score. The Weber fraction is the JND divided by the stimulus intensity (real spring stiffness). The literature sometimes assimilates the Weber fraction to JND, as we will in this paper. It will be given as a percentage of the reference torsion spring stiffness. The PSE is calculated for a proportion of $50 \%$ of "virtual spring is stiffer" answers, which is interpreted as random answers ( 0.5 probability for either answer). In this paper we will consider the PSE as a subjective distortion (or error) of perception, but this doesn't mean that we assume a one-to-one correspondence in the relation between perceived and actual stiffnesses.

Some subjects always answered that the virtual spring is stiffer; we will refer to these subjects as "purely haptic". The ratio of purely haptic subjects was $2 / 17$ for the isometric device, and 6/17 for the elastic device. We chose not to consider these subjects in JND and PSE analysis.

Z-score transformation of the psychometric function, on which the calculation of JND and PSE is based, is shown

\footnotetext{
${ }^{1}$ See definition of JND and PSE in Section 2
}

in Figure 8 Figure 9 shows the JND and the PSE for both experiments. For the isometric device, values of JND were: $28.2 \%$ for spring 1 and $14.2 \%$ for spring 2 . The PSE value for which the virtual spring is perceived as equal to the real spring is $P S E_{1}=2.11 \cdot 10^{-2} \mathrm{~N} \cdot \mathrm{m} \cdot \mathrm{rad}^{-1}$ for spring 1 and $P S E_{2}=2.75 \cdot 10^{-2} \mathrm{~N} \cdot \mathrm{m} \cdot \mathrm{rad}^{-1}$ for spring 2. This corresponds to subjective distortion of perceptions of $+2.8 \%$ and $-14 \%$ of the real spring values as shown in Figure 9. For the elastic device, values of JND were respectively $9.1 \%$ and $4.4 \%$ for springs 1 and 2 . PSE values were $P S E_{1}=1.59 \cdot 10^{-2} \mathrm{~N} \cdot \mathrm{m} \cdot \mathrm{rad}^{-1}$ for the spring 1 simulation and $P S E_{2}=2.70 \cdot 10^{-2} \mathrm{~N} \cdot \mathrm{m} \cdot \mathrm{rad}^{-1}$ for spring 2 . These values correspond to a subjective distortion of perception of $-22.5 \%$ and $-15.36 \%$ relative to the real spring stiffness (Figure 9).

Applied Torque. The obtained torque vs. time curve is a succession of peaks (local maximum values of torque). The number of peaks for each comparison was calculated. Average number of peaks is $4.78(\sigma=0.83)$ for the isometric device and $4.84(\sigma=1.41)$ for the elastic device. This value seems not different between type of device, nor between comparison spring values. Peak values shows important difference between isometric and elastic devices. Figure 6 shows average peak values and standard deviation of peak values within each comparison, for each device type.

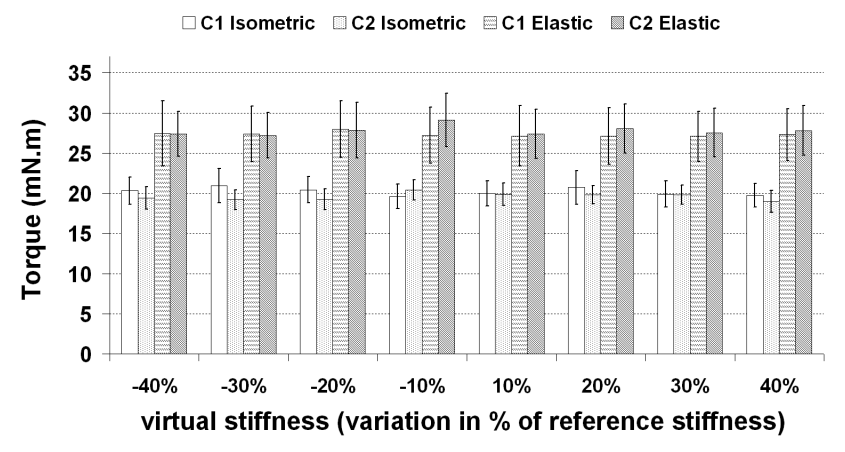

Figure 6. Average of torque peaks for each comparison between the two real springs (C1 and $\mathrm{C2}$ stiffnesses) and the presented virtual springs, with standard deviation bars.

Answer time. Figure 7 shows answer times for both isometric and elastic modes.

Perceived displacement of the device. In the isometric device group, 13 persons were subject to a displacement illusion, since they reported a displacement of the static cap. We emphasize here that the subjects could not see the 


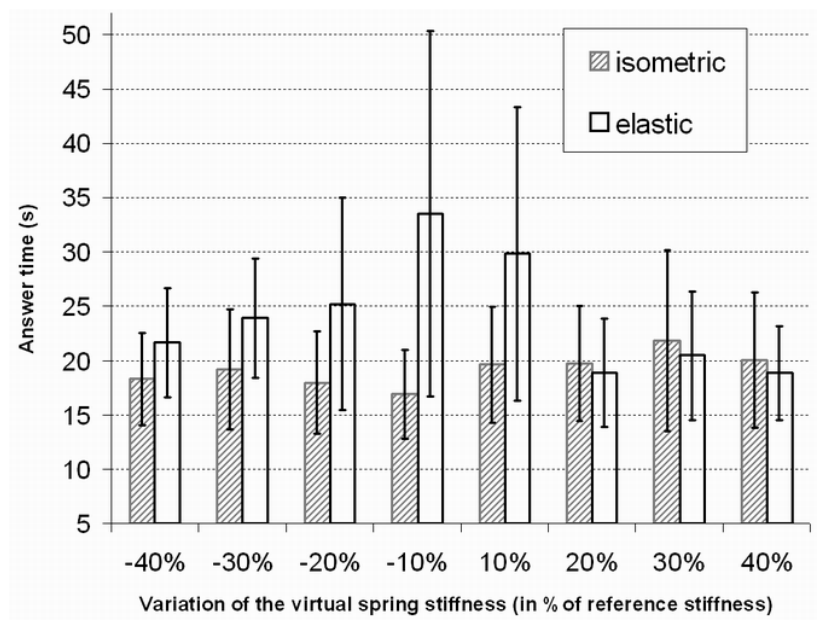

Figure 7. Answer time for isometric and elastic mode (with standard deviation bars).

\begin{tabular}{|l|l|l|l|}
\hline & $\begin{array}{l}\text { Static strat- } \\
\text { egy }\end{array}$ & $\begin{array}{l}\text { Dynamic } \\
\text { strategy }\end{array}$ & $\begin{array}{l}\text { Combination } \\
\text { of strategies }\end{array}$ \\
\hline Isometric & $11 / 17$ & $4 / 17$ & $2 / 17$ \\
& $(64,7 \%)$ & $(23,5 \%)$ & $(11,8 \%)$ \\
\hline Elastic & $12 / 17$ & $3 / 17$ & $2 / 17$ \\
& $(70,6 \%)$ & $(17,6 \%)$ & $(11,8 \%)$ \\
\hline
\end{tabular}

Table 1. Subject's strategy: Ratio of answers for each device type (17 subjects per device)

plastic cap, as it was intentionally hidden by a cardboard cover. The average perceived rotation of the device was $27.2^{\circ}\left(\sigma=25.6^{\circ}\right)^{2}$ for all the subjects. For the elastic device group, the perceived rotation of the input device was $59.3^{\circ}\left(\sigma=17.7^{\circ}\right)$. This also corresponds to a displacement illusion, since the maximum elastic device rotation was $22^{\circ}$, which corresponds to the maximum $90^{\circ}$ allowed rotation of the virtual cap.

User strategy. Subjects reported having three strategies: A "static" strategy, in which they compared stiffnesses by estimating the torque for a fixed angle, a "dynamic" strategy, involving several back-and-forth movements without paying much attention to the terminal displacement, and finally some users reported using a combination of these strategies. Table 1 reports the results for user strategy.

\footnotetext{
${ }^{2}$ Standard deviation
}

\section{Discussion}

Results show that subjects were able to compare real and simulated torque forces with both devices.

Performance varies depending on input device type: subjects discriminated the real and virtual springs with a better resolution when using an elastic device than with the isometric device, on the basis of JND results. Since these are stiffness JNDs, we cannot compare them to the torque JND $(12,7 \%)$ found in [13]. The movements of the fingers and hands are more important with the elastic device, this can give the user a better proprioceptive sense. Internal stiffness of the elastic device and rotation can give the user information relative to his mechanical work. These two types of information can explain a better performance with the elastic device. Tan et al. [18] found that the existence of mechanical work information increases the user resolution in stiffness discrimination tasks, and that proprioception is likely to provide such information. On the basis of PSE values shown in Figure 9, we can observe that there is an overestimation of the virtual springs for the elastic device. This phenomenon appears with the isometric device too, but only for the stiffer spring comparisons. This could be explained by the measured average torque (peak values) applied on the device, which is systematically higher with the elastic device.

The maximum applied torque seems not to depend on the simulated stiffness; moreover, it seems almost constant over the different simulated springs. This result shows that the successful discrimination between real and pseudo-haptic simulated springs does not directly rely on actual forces, nor on actual mechanical work, but relies more on a haptic illusion.

This haptic illusion could occur with respect to perceived terminal force or perceived mechanical work. As a hypothesis, we propose that the subjects may use a "perceived mechanical work" which combines perceived displacement of the device and applied torque. Further work is needed to address this hypothesis: it would be interesting to study the perceived displacement of the device as a function of simulated stiffness.

We carried out an ANOVA on answer time with repeated measures. Analysis is done with two within-subjects factors: 1) Presented virtual stiffness, as a percentage of reference stiffness and 2) reference stiffness, and one betweensubjects factor: device type, isometric or elastic. Results show a non-significant effect of device type on answer time $(\mathrm{F}(1,24)=3.889, \mathrm{p}=0.06)$, with a tendency of longer answer times with the elastic device (average 24.04s, and $19.21 \mathrm{~s}$ for isometric). There is no significant influence of reference stiffness nor presented virtual stiffness.

There is a significant two-way interaction between device type and presented virtual stiffness $(\mathrm{F}(7,168)=2,88$ 

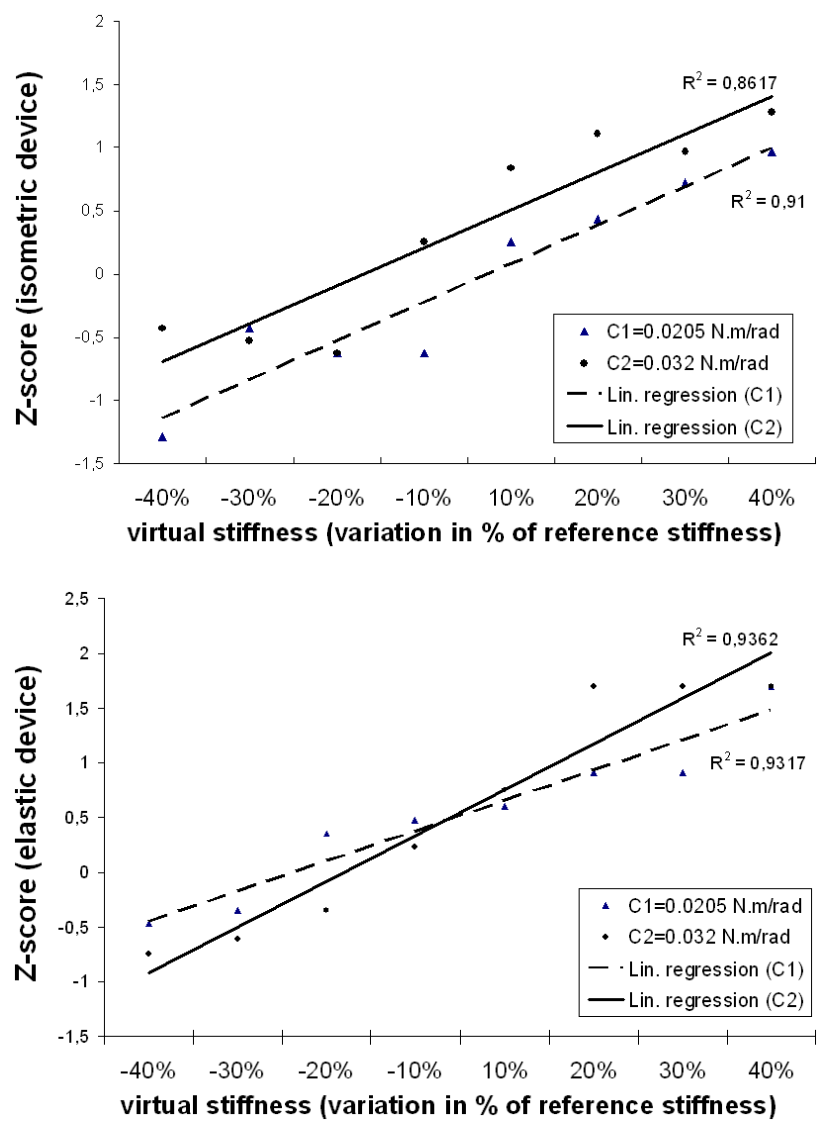

Figure 8. Z-transformation of psychometric function for isometric and elastic devices.

$(\mathrm{p}<0.007))$. Answer time seems not to be affected by variations of virtual stiffness with the isometric device, but it is affected with the elastic device: the smaller the difference between real and virtual springs, the longer the answer time. It seems that the difficulty of the discrimination increases when the difference between the real and the virtual springs decreases for the elastic device. This phenomenon resembles the real situation, whereas difficulty seems constant over the comparisons for the isometric device. This could characteristic of a more realistic feedback for the elastic device.

In both experiments, subjects experienced an illusion phenomenon. It is difficult to quantitatively compare the magnitudes of these two illusions since their nature is different: for the isometric device there is an illusion of displacement while there is no actual displacement of the device, whereas for the elastic device the illusion leads to an overestimation of the actual displacement. Qualitatively, we can consider that perceiving a displacement where there is none is a greater illusion. This can be interpreted as a com-
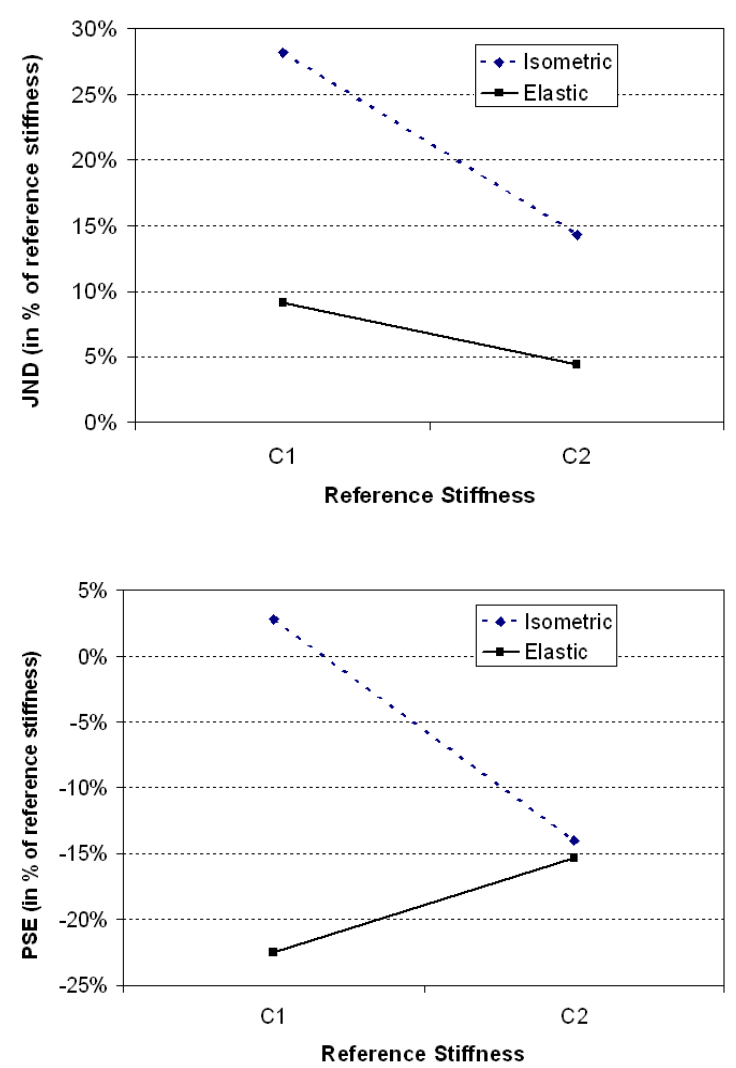

Figure 9. Just Noticeable Differences and Point of Subjective Equality, on virtual springs, for each real -reference- spring stiffness $\mathrm{C} 1$ and $\mathrm{C2}$.

pensation for the lack of actual proprioceptive feedback. The strategy reported by the users is not significantly different between the two devices. Conversely, on the basis of torque recordings, the actual behavior of users seems to be a more "dynamic" strategy: there are about 5 peaks of applied torque for all comparisons, and an average peak duration (including increase and decrease time) of $0.35 \mathrm{~s}(\sigma=0.075)$ for the isometric and $0.81 \mathrm{~s}(\sigma=0.17)$ for the elastic device. This reinforces the hypothesis that a mechanical work cue is used, based on a series of turns on the device. There were two haptic-oriented subjects (whose answers are always "the virtual spring is stiffer") for the isometric device, and six for the elastic device. It isn't possible to conclude that this difference is significant on the basis of our current data $\left(\chi^{2}=2.615, \mathrm{n} . \mathrm{s}\right)$. If confirmed, such a tendency could be due to the more realistic haptic behavior of the virtual spring with the elastic device, leading to a higher proportion of "physically correct" answers that the virtual spring is always stiffer. 


\section{Conclusion}

In this work, we investigated whether pseudo-haptic feedback is suitable for simulating torque. Experimental results show that users were able to discriminate pseudohaptic simulated torsion springs and real springs. Results for perception resolution (JND) showed better performance when using an elastic input device compared to an isometric one. The elastic device yields a better resolution but a higher subjective distortion of perception compared to the isometric device. On the basis of applied torque measurements, we proposed the hypothesis that a cue called "perceived mechanical work" is used during the discrimination.

Applications such as surgical training for flexible endoscopy could gain from pseudo-haptic torque feedback, as the user could perceive forces from a virtual endoscope tip through navigation wheels used to control the endoscope. Another possible application would be training on a virtual lathe, where the user controls the cutting of a piece of wood with control wheels (as in the real situation). Pseudo-haptic torque feedback through control wheels would give him information about the forces applied on the piece of wood, and train him to avoid its breaking.

Future work will deal with integrating torque pseudohaptic feedback in an immersive virtual environment such as a responsive workbench. Haptic feedback could be performed with a tracked hand-held or wearable system, enabling the user to test mechanical properties of objects in the environment.

\section{Acknowledgements}

This research was funded by the Regional Council of Ilede-France and the French Ministry of Research within the RNTL Perf-RV project.

\section{References}

[1] Gyro moment haptic interface, 2002. http://intron.kz. tsukuba.ac.jp/vrlab_web/gyro/gyro_j.html, University of Tsukuba, VR Lab.

[2] Haption, 2002. http://www.haption.com.

[3] Mpb technologies, 2002. http://www.mpb-technologies.ca /space/freedom6_2000/f6s/freedom6s.html.

[4] Sensable technologies, 2002. http://www.sensable.com.

[5] C. Basdogan, C. Ho, M. Srinivasan, and M. Slater. An experimental study on the role of touch in shared virtual environments. In ACM Transactions on Computer-Human Interation, volume 7, December 2000.

[6] C. Basdogan, C.-H. Ho, and S. M. A. Virtual environments in medical training: Graphical and haptic simulation of laparoscopic common bile duct exploration. In IEEE/ASME Transactions on Mechatronics, volume 6, pages 269-285, 2001.
[7] P. Berkelman and R. Hollis. Interacting with virtual environments using a magnetic levitation haptic interface. In IEEE/RSJ Intelligent Robots and Systems Conference, Aug 1995.

[8] F. Biocca, J. Kim, and Y. Choi. Visual touch in virtual environments: An exploratory study of presence, multimodal interfaces and cross-modal sensory illusions. In Presence, volume 10, pages 247-265. MIT, 2001.

[9] Y. Fukui, K. Nakata, N. Nakamura, and J. Yamashita. Handheld torque feedback device. In Conference Abstracts and Applications of ACM SIGGRAPH, page 192, 2001.

[10] G. Gescheider. Psychophysics Method, Theory, and Application. Lawrence Elbaum Associates, New Jersey, US, 1985.

[11] S. Grange, F. Conti, P. Helmer, P. Rouiller, and C. Baur. Overview of the delta haptic device. In Eurohaptics 2001 Conference Proceedings, Birmingham UK, 2001.

[12] C.-H. Ho, C. Basdogan, and M. A. Srinivasan. Ray-based haptic rendering: force and torque interactions between a line probe and $3 \mathrm{~d}$ objects in virtual environments. In International Journal of Robotics Research, volume 19(7), pages 668-683, 2000.

[13] L. Jandura and M. Srinivasan. Experiments on human performance in torque discrimination and control. In Dynamic Systems and Control, ASME, DSC-vol.55-1, C. J. Radcliffe, (ed.), 1994.

[14] A. Lecuyer, J.-M. Burkhardt, S. Coquillart, and P. Coiffet. Boundary of illusion : an experiment of sensory integration with a pseudo-haptic system. In Proceedings of IEEE VR'2001, 2001.

[15] A. Lecuyer, S. Coquillart, A. Kheddar, P. Richard, and P. Coiffet. Pseudo-haptic feedback: Can isometric input devices simulate force feedback? In Proceedings IEEE $V R$ '2000, pages 83-90, 2000.

[16] T. Massie and K. Salisbury. The phantom haptic interface: A device for probing virtual objects. In ASME Winter Annual Meeting , DSC., volume 55-1, pages 295-300, 1994.

[17] E.-L. Sallnäs, K. Rassmus-Gröhn, and C. Sjöström. Supporting presence in collaborative environments by haptic force feedback. ACM Transactions on Computer-Human Interaction (TOCHI), 7(4):461-476, 2000.

[18] H. Tan, N. Durlach, Y. Shao, and M. Wei. Manual resolution of compliance when work and force cues are minimized. In Kazerooni H., Colgate J. E., Adelstein B. D. (Eds.), ASME, DSC-49, pages 99-104, 1993.

[19] S. Zhai. Investigation of feel for 6DOF inputs: Isometric and elastic rate control for manipulation in 3D environments. In Proceedings of the Human factors and Ergonomics Society 37th annual meeting, 1993. 